\title{
Analysis of an SERIV Epidemic Model of Human Influenza
}

\author{
Lijuan Zhang, Yibin Zhao and Fuchang Wang \\ Basic Courses Department, Institute of Disaster Prevention, Sanhe, Hebei Province, 065201, China
}

\begin{abstract}
In this present paper a model with media coverage and vaccination is proposed on the spread of H7N9 influenza. We worked out the disease-free equilibrium and basic reproductive number by mathematical method and analyzed the sensitivity and uncertainty of the basic reproductive number by random sampling. At the same time, we also make some simulation on the stability of the disease-free equilibrium and the influence of the media coverage on the spread of the disease. These conditions can be used to gain important insights into the effect of media coverage and treatment on the prevention and control of influenza. The simulations are also conducted to confirm and extend the analytic results.
\end{abstract}

Keywords- influenza H7N9; media coverage; vaccination; stability

\section{INTRODUCTION}

Mathematical modeling in epidemiology has become a more and more useful tool in the analysis of spread and control of infectious diseases. There are diseases such as SARS and flu that exhibit some distinct features such as rapid spatial spread and visible symptoms [1]. H7N9 is one of them, and it is a new subtype H7N9 virus infection in eastern China in 2013. It has posed potential threat to the health of the global human being. Up to now, the cases are distributed. There is no evidence of human-to-human transmission (see figure 1).

Comparative knowledge of the effectiveness and efficacy of different control strategies is necessary to design useful influenza control programs. Mathematical modeling of the spread of influenza can play an important role in comparing the different control strategies [2-5]. Although different control and prevention strategies are available to control influenza transmission, influenza has been a major cause of morbidity and mortality among humans all over the world. The models currently have provided useful information about the impact of various control measures in the disease dynamics, for example: vaccination or antiviral use [6]. In addition the basic reproduction ratio or basic reproductive rate or basic reproduction number is one of the most important theoretical concepts in theoretical infectious diseases epidemiology[7].

Media reporting plays a key role in the perception, management and even creation of crisis [8]. Since media reports are retrievable and because the messages are widely distributed, they gain authority as an inter-subjective anchorage for personal recollection [9]. At times of crisis, non-state-controlled media thrive, while state-controlled media are usually rewarded for creating an illusion of normalcy [10].
The role of media coverage on disease outbreaks in thus crucial and should be given prominence in the study of disease dynamics. However, these models now have seldom considered the latent period or the effect of media coverage. As we know that the influenza indeed has a latent period before the infected become ill. The main aim of this study is to investigate the impact of media coverage on the spread and control of an influenza strain when there is a latent period and a vaccine is available, and where the media reporting of both disease dynamics and vaccination is high. Reporting the number of individuals who vaccinate may have a positive effect on the disease transmission by increasing the vaccination rate.

In this article, we established a model with the latency effects on the basis of [6], and analyzed the stability of the model. From the simulation about the factors we obtain some control strategies. At last, we discussed the sensitivity of the basic reproduction number, which will help us to determine the relative importance of different parameters in transmission and prevalence of influenza.

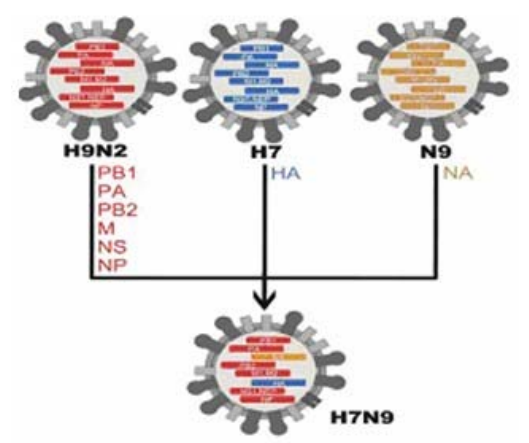

FIGURE I. THE FORMULATION OF THE H7N9

\section{MODEL FRAMEWORK}

We divide the population $N$ into five sub-populations, according to their disease status: susceptible $E$, vaccinated $V$, latent $E$, infected $I$, and recovered $R$. Our model monitors the dynamics of influenza based on a single strain without effective cross-immunity against the strain. The susceptible population is increased by recruitment of individuals (either by birth or immigration), and by the loss of immunity (acquired through previous vaccination or natural) infection. The model is represented by the following system of ordinary differential equations: 


$$
\left\{\begin{array}{l}
\frac{\mathrm{d} S}{\mathrm{dt}}=\Lambda+\omega \mathrm{V}-(\theta+\mu) \mathrm{S}-\left(\beta_{1}-\beta_{2} \frac{\mathrm{I}}{\mathrm{m}_{\mathrm{I}}+I}\right) \mathrm{SI}+\sigma \mathrm{R} \\
\frac{\mathrm{d} E}{\mathrm{dt}}=\left(\beta_{1}-\beta_{2} \frac{\mathrm{I}}{\mathrm{m}_{\mathrm{I}}+I}\right) \mathrm{SI}+\left(\beta_{1}-\beta_{3} \frac{\mathrm{I}}{\mathrm{m}_{\mathrm{I}}+I}\right)(1-\gamma) \mathrm{VI}-(\mu+\eta) \mathrm{E} \\
\frac{\mathrm{d} I}{\mathrm{dt}}=\eta \mathrm{E}-(\mu+\alpha+\lambda) \mathrm{I} \\
\frac{\mathrm{d} V}{\mathrm{dt}}=\theta \mathrm{S}-(\mu+\omega) \mathrm{V}-\left(\beta_{1}-\beta_{3} \frac{\mathrm{I}}{\mathrm{m}_{\mathrm{I}}+I}\right)(1-\gamma) \mathrm{VI} \\
\frac{\mathrm{d} R}{\mathrm{dt}}=\lambda \mathrm{I}-(\mu+\sigma) \mathrm{R}
\end{array}\right.
$$

The biological meaning of the parameters and chosen values of these parameters are speci fied in the Table 1 .

We suppose that the recovered individuals can not be vaccinated. Also, a vaccinated who gets infected and those recovers will return to the susceptible class with no vaccine protection. This is true even if $\omega$ is quite small but $\sigma$ and $\lambda$ are large. For example, if vaccination lasts three years, but recovery and loss of immunity takes 6months, then we are assuming this person is subsequently unvaccinated. The rate at which information is spread by the media rises as infectives increase, but eventually levels off at a plateau (or asymptote) at which the information (rate) remains constant. Our model is clearly a crude reflection of the complicated nonlinear phenomena of the transmission dynamics, and it does not incorporate the self-control property due to the change of

\begin{tabular}{|c|c|c|}
\hline Parameter & Description & Value \\
\hline$\Lambda$ & $\begin{array}{l}\text { The rate at which individuals are } \\
\text { recruited into population }\end{array}$ & $7.14 \times 10^{-8}$ \\
\hline$\theta$ & $\begin{array}{l}\text { The rate at which susceptible individuals } \\
\text { receive vaccine }\end{array}$ & 0.002 \\
\hline$\mu$ & $\begin{array}{l}\text { The rate at which people leave the } \\
\text { population (natural death or emigration) }\end{array}$ & $5.5 \times 10^{-7}$ \\
\hline$\beta_{1}$ & $\begin{array}{c}\text { The rate at which susceptibles get } \\
\text { infected }\end{array}$ & 0.514 \\
\hline$\omega$ & $\begin{array}{l}\text { The rate at which vaccine-based } \\
\text { immunity wanes }\end{array}$ & 0.015 \\
\hline$\gamma$ & The vaccine efficacy & 0.1 \\
\hline$\alpha$ & The death rate due to the infection & 0.01 \\
\hline$\lambda$ & The recovery rate from infection & 0.2 \\
\hline$\eta$ & The mean duration of latency & 0.5 \\
\hline $\begin{array}{c}\beta_{2} \frac{\mathrm{I}}{\mathrm{m}_{\mathrm{I}}+I} \\
\beta_{3} \frac{\mathrm{I}}{\mathrm{m}_{\mathrm{I}}+I}\end{array}$ & $\begin{array}{l}\text { The effect of reduction of the contact } \\
\text { rate when infectious and vaccinated } \\
\text { individuals are reported in the media } \\
{[1][11][12]}\end{array}$ & $\begin{array}{l}\beta_{2}=0.013 \\
\beta_{3}=0.015\end{array}$ \\
\hline $\mathrm{m}_{\mathrm{I}}$ & $\begin{array}{l}\text { The impact of media coverage on the } \\
\text { contact transmission }\end{array}$ & $\mathrm{m}_{\mathrm{I}}=-0.9$ \\
\hline$\sigma$ & Duration of immunity loss & $1 / 365$ \\
\hline$\frac{\mathrm{I}}{\mathrm{m}_{\mathrm{I}}+I}$ & $\begin{array}{l}\text { A continuous bounded function which } \\
\text { takes into account disease saturation or } \\
\text { psychological effects[13] }\end{array}$ & \\
\hline
\end{tabular}
avoidance patterns of individuals at different stages of the infectious process[1]

TABLE I. MODEL PARAMETERS AND THEIR INTERPRETATIONS

News coverage may have a significant impact on avoidance behaviors at both individual and society levels, which may reduce the effective contact between susceptible and infectious individuals; we include this via a saturation incidence functional response.

It is easy to see that the model will be studied in the following region:

$$
\Omega=\left\{(S, E, I, V, R) \in R_{+}^{5}\right\}
$$

Which is positively invariant and attracting (thus, the model is mathematically and epidemi-ologically well-posed); it is therefore sufficient to consider solution in $\Omega$. Existence, uniqueness and continuation results for model system (2.1) hold in this region and all solutions of this system starting in $\Omega$ remain in $\Omega$ for all $t \geqslant 0$.

\section{STABILITY OF THE EQUILIBRIUM STATES}

The disease-free equilibrium of the system is given by

$$
\left(\frac{\Lambda(\mu+\omega)}{\mu(\theta+\mu+\omega)}, 0,0, \frac{\Lambda \theta}{\mu(\theta+\mu+\omega)}, 0\right)
$$

The endemic equilibrium of the system is given by $\left(S^{*}, E^{*}\right.$, $\left.I^{*}, V^{*}, R^{*}\right)$, which satisfied $S^{*}, E^{*}, I^{*}, V^{*}, R^{*}>0$, and

$$
\begin{aligned}
& h_{1}(I)=\left(\beta_{1}-\beta_{2} \frac{I}{m_{I}+I}\right) I \\
& h_{2}(I)=\left(\beta_{1}-\beta_{3} \frac{I}{m_{I}+I}\right)(1-\gamma) I \\
& S^{*}=\frac{\left(\mu+\omega+h_{2}\left(I^{*}\right)\right)(\mu+\eta)(\mu+\alpha+\lambda) I^{*}}{\eta\left[h_{1}\left(I^{*}\right)\left(\mu+\omega+h_{2}\left(I^{*}\right)\right)+h_{2}\left(I^{*}\right) \theta\right]} \\
& E^{*}=\frac{\mu+\alpha+\lambda}{\eta} I^{*} \\
& V^{*}=\frac{(\mu+\eta)(\mu+\alpha+\lambda) \theta I^{*}}{\eta\left[h_{1}\left(I^{*}\right)\left(\mu+\omega+h_{2}\left(I^{*}\right)\right)+h_{2}\left(I^{*}\right) \theta\right]} \\
& R^{*}=\frac{\lambda}{\mu+\sigma} I^{*}
\end{aligned}
$$

$I^{*}$ is the root of the equation: $\Lambda+\omega V^{*}-(\theta+\mu) S^{*}-h_{1}\left(I^{*}\right) S^{*}$ $+\sigma R^{*}=0$, and this is a equation about $I^{*}$.

The basic reproductive ratio $R_{0}$ is defined as the expected number of secondary infections caused by an infective individual upon entering a totally susceptible population. This quantity is not only important in describing the infectious power of the disease, but can also supply information for controlling the spread of the disease [14]. The stability of the equilibrium is governed by the basic reproductive ratio $R 0$, using the next-generation method[15], we have 
$X=(S, V, R), Y=(E), Z=(I), U_{0}=\left(\frac{\Lambda}{\mu}, 0,0\right), \widetilde{g}\left(X^{*}, X\right)=\frac{h_{1}(I) S+h_{2}(I) V}{\mu+\eta}$ $A=D_{Z} h\left(X^{*}, \widetilde{g}\left(X^{*}, 0\right), 0\right)$

$=D_{Z}\left[\begin{array}{l}\eta \frac{\left(\beta_{1}-\beta_{2} \frac{I}{m_{I}+I}\right)}{\mu+\eta} \frac{\Lambda(\mu+\omega)}{\mu(\theta+\mu+\omega)} I \\ +\frac{\eta}{\mu+\eta}\left(\beta_{1}-\beta_{3} \frac{I}{m_{I}+I}\right)(1-\gamma) \frac{\Lambda \theta}{\mu(\mu+\theta+\omega)}-(\mu+\alpha+\lambda) I\end{array}\right]$ $=\frac{\beta_{1} \eta}{\mu+\eta} \frac{\Lambda(\mu+\omega)}{\mu(\theta+\mu+\omega)}+\beta_{1} \frac{\eta}{\mu+\eta}(1-\gamma) \frac{\Lambda \theta}{\mu(\mu+\theta+\omega)}-(\mu+\alpha+\lambda)$ $=\frac{\beta_{1} \eta \Lambda[\mu+\omega+\theta(1-\gamma)]}{(\mu+\eta) \mu(\mu+\theta+\omega)}-(\mu+\alpha+\lambda)$

Thus we have

$$
\begin{aligned}
& M=\frac{\beta_{1} \eta \Lambda[\mu+\omega+\theta(1-\gamma)]}{(\mu+\eta) \mu(\mu+\theta+\omega)} \\
& D=\mu+\alpha+\lambda \\
& R_{0}=M D^{-1}=\frac{\beta_{1} \eta \Lambda[\mu+\omega+\theta(1-\gamma)]}{(\mu+\eta) \mu(\mu+\theta+\omega)(\mu+\lambda+\alpha)}
\end{aligned}
$$

Local stability of the disease-free equilibrium is determined by the following lemma

Lemma 3.1 The disease-free equilibrium is locally asymptotically stable if $R_{0}<1$, and unstable if $R_{0}>1$.

Proof The Jacobian of the system evaluated at the disease-free equilibrium is given by

$J_{I=0}=\left[\begin{array}{ccccc}-(\theta+\mu) & 0 & -\frac{\beta_{1} \Lambda(\mu+\omega)}{\mu(\theta+\mu+\omega)} & \omega & \sigma \\ 0 & -(\mu+\eta) & \frac{\beta_{1} \Lambda[\mu+\omega-(1-\gamma) \theta]}{\mu(\mu+\theta+\omega)} & 0 & 0 \\ 0 & \eta & -(\mu+\lambda+\alpha) & 0 & 0 \\ \theta & 0 & -\frac{\beta_{1}(1-\lambda) \Lambda \theta}{\mu(\mu+\theta+\omega)} & -(\mu+\omega) & 0 \\ 0 & 0 & \lambda & 0 & -(\mu+\sigma)\end{array}\right]$

The eigenvalues of $J_{I=0}$ are $\xi_{1}={ }_{-}(\mu+\sigma), \xi_{2}={ }_{-}(\theta+\mu), \xi_{3}=$ $-(\mu+\omega), \xi_{4}, \xi_{5}$ are the root of the equation

$$
\mathrm{m}^{2}+\mathrm{m}(2 \mu+\alpha+\lambda)+\mu(\mu+\alpha+\lambda)-\frac{\eta \beta_{1} \Lambda[\mu+\omega-(1-\gamma) \theta]}{\mu(\mu+\theta+\omega)}=0
$$

Then we have

$$
\begin{aligned}
& m_{4}+m_{5}=-(2 \mu+\alpha+\lambda) \\
& m_{4} m_{5}=\mu(\mu+\alpha+\lambda)-\frac{\eta \beta_{1} \Lambda[\mu+\omega-(1-\gamma) \theta]}{\mu(\mu+\theta+\omega)}
\end{aligned}
$$

For the reason $R_{0}<1$, we can conclude that $m_{4} m_{5}>0$, thus $m_{4}<0, m_{5}<0$.
The local stability of the disease-free equilibrium is that all the eigenvalues be negative. From above we can conclude that the disease-free equilibrium is locally asymptotically stable.

In the following, we will prove the global stability of the disease-free equilibrium. We adopt the method of CastilloChavez et al [16] and we rewrite the set of model equations in the form

$$
\frac{d X_{G}}{d t}=F\left(X_{G}, Z_{G}\right) \quad \frac{d Z_{G}}{d t}=G\left(X_{G}, Z_{G}\right)
$$

With $G\left(X_{G}, 0\right)=0, X_{G} \in R^{3}$ denotes the number of uninfected classes and $Z_{G} \in R^{2}$ denotes the number of infected classes. $U 0 G=\left(X_{G}^{*}, 0\right)$ denotes the disease-free equilibrium of the system where

$$
X_{G}^{*}=\left(\frac{\Lambda(\mu+\omega)}{\mu(\theta+\mu+\omega)}, \frac{\Lambda \theta}{\mu(\theta+\mu+\omega)}\right) \text {. }
$$

For the set of equations in (2.1), we set $X_{G}=(S, V, R)$ and $Z_{G}=(E, I)$. The conditions $H_{1}, H_{2}$ below must be met for global stability.

$$
H_{1}: \operatorname{For} \frac{d X_{G}}{d t}=F\left(X_{G}, 0\right), X_{G}^{*} \text { is globally }
$$

asymptotically stable.

$$
H_{2}: G\left(X_{G}, Z_{G}\right)=A_{G} Z_{G}-\hat{G}\left(X_{G}, Z_{G}\right)
$$
$\hat{G}\left(X_{G}, Z_{G}\right) \geqslant 0$ for $\left(X_{G}, Z_{G}\right) \in \Omega$, where $A_{G}=D_{Z G}\left(X_{G}^{*}, 0\right)$ is M-matrix(the off-diagonal elements of $A$ are nonnegative) and $\Omega$ is the region where the model makes biological sense.

If the above two conditions are satisfied, then the following theorem holds.

Theorem 3.2 (Castillo-Chavez et al [6]) The fixed point $U_{0 G}=\left(X_{G}^{*}, 0\right)$ is a globally stable equilibrium of (2.1) provided that and that assumptions are satisfied.

\section{SENSITIVITY ANALYSIS}

\section{A. Simulation of the Disease-Free Equilibrium}

First we study the stability of the disease-free equilibrium. We obtain using parameter data of table 1 and the formula (3.1). The simulation of the disease-free equilibrium stability is shown by Fig. 2. 


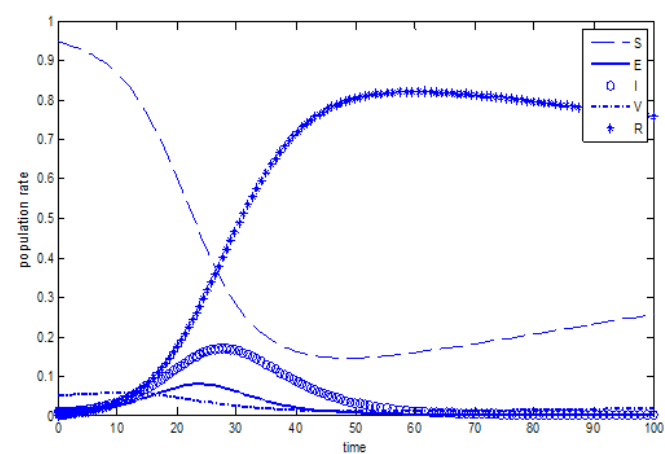

FIGURE II. THE STABILITY OF THE DISEASE-FREE EQUILIBRIUM

\section{B. Sensitivity Indices of $R_{0}$ Based on Perturbation of Five Point Estimations}

Next we discuss the sensitivity indices of $R 0$ based on perturbation of fixed point estima- tions. In the next section, sensitivity analysis is performed to identify the parameters which are important in contributing variability in the outcome of the basic reproduction number based on their estimation uncertainty. Partial rank correlation (PRCCs) is the method used here to find statistical influence of any parameters and $R_{0}$ [19], especially the monotonicity i.e. how well the relationship between the parameters and the basic reproduction number $R_{0}$ varies. The effect of variability of the input parameter on the outcome parameter using variable factor prioritization method by reduction of variance i.e. sensitivity index has been used here [17]. The larger the partial rank correlation coefficient, the larger influence of the input parameter is on magnitude of $R_{0}$.

The basic reproduction number $R_{0}$ is a function of 9 parameters where

$$
R_{0}=\frac{\beta_{1} \eta \Lambda[\mu+\omega+\theta(1-\gamma)]}{(\mu+\eta) \mu(\mu+\theta+\omega)(\mu+\lambda+\alpha)}
$$

A small perturbation $\delta_{\lambda}$ to a parameter $\lambda$ and the corresponding change in $R_{0}$ is as $\delta_{R_{0}}$ is given by

$\delta_{R_{0}}=R_{0}\left(\lambda+\delta_{\lambda}\right)-R_{0}(\lambda)=\frac{R_{0}\left(\lambda+\delta_{\lambda}\right)-R_{0}(\lambda)}{\delta_{\lambda}} . \delta_{\lambda} \approx \delta_{\lambda} \frac{\partial R_{0}}{\partial \lambda}$.

Then the normalized sensitivity index $\varphi_{\lambda}$ is defined as

$$
\varphi_{\lambda}=\frac{\delta R_{0}}{R_{0}} \cdot \frac{\delta \lambda}{\lambda}=\frac{\lambda}{R_{0}} \cdot \frac{\partial R_{0}}{\partial \lambda} .
$$

Thus the normalized sensitivity indices for 9 parameters are obtained as

$$
\frac{\beta_{1}(\mu+\eta) \mu(\mu+\theta+\omega)(\mu+\lambda+\alpha)}{\beta_{1} \eta \Lambda[\mu+\omega+\theta(1-\gamma)]} \cdot \frac{\eta \Lambda[\mu+\omega+\theta(1-\gamma)]}{(\mu+\eta) \mu(\mu+\theta+\omega)(\mu+\lambda+\alpha)}=1
$$

As the same method we can obtain $\varphi_{\lambda}=1$.

$$
\begin{aligned}
& \varphi_{\mu}=\frac{\mu}{R_{0}} \cdot \frac{\partial R_{0}}{\partial_{\mu}} \\
& =\mu \beta\left[\frac{1}{\mu+\omega+\theta(1-\gamma)}+\frac{1}{\mu+\eta}+\frac{1}{\mu}+\frac{1}{\mu+\theta+\omega}+\frac{1}{\mu+\lambda+\alpha}\right] \\
& \varphi_{\omega}=\frac{\omega}{R_{0}} \cdot \frac{\partial R_{0}}{\partial_{\omega}} \\
& =\frac{\omega(\mu+\eta) \mu(\mu+\theta+\omega)(\mu+\lambda+\alpha)}{\beta_{1} \eta \Lambda[\mu+\omega+\theta(1-\gamma)]} \cdot \frac{\beta_{1} \eta \Lambda}{(\mu+\eta) \mu(\mu+\lambda+\alpha)} \\
& \frac{(\mu+\theta+\omega)-(\mu+\omega+\theta(1-\gamma))}{(\mu+\theta+\omega)^{2}} \\
& =\omega\left[\frac{1}{\mu+\omega+\theta(1-\gamma)}-\frac{1}{\mu+\theta+\omega}\right] \\
& \varphi_{\theta}=\theta\left(\frac{1-\gamma}{\mu+\omega+\theta(1-\gamma)}-\frac{1}{\mu+\theta+\omega}\right) \\
& \varphi_{\gamma}=\frac{\gamma}{R_{0}} \cdot \frac{\partial R_{0}}{\partial_{\gamma}}=\frac{-\lambda}{\mu+\lambda+\alpha} \\
& \varphi_{\eta}=\frac{\eta}{R_{0}} \cdot \frac{\partial R_{0}}{\partial_{\eta}}=\frac{\mu}{\mu+\eta} \\
& \varphi_{\alpha}=\frac{\alpha}{R_{0}} \cdot \frac{\partial R_{0}}{\partial_{\alpha}}=\frac{-\alpha}{\mu+\lambda+\alpha}
\end{aligned}
$$

We can get the table 2 by mathematical calculation

TABLE II. SENSITIVITY INDICES OF $\mathrm{R}_{0}$

\begin{tabular}{ccc}
\hline $\begin{array}{c}\text { Parameter } \\
\text { Sensitivity } \\
\text { indices of } R_{0}\end{array}$ & Value & \\
\hline$\Lambda$ & $\varphi_{\Lambda}=+1.000000000$ & $7.14 \times 10^{-8}$ \\
$\theta$ & $\varphi_{\theta}=-0.021898571867231$ & 0.002 \\
$\mu$ & $\varphi_{\mu}=0.514035367290174$ & $5.5 \times 10^{-7}$ \\
$\beta_{1}$ & $\varphi_{\beta_{1}}=+1.000000000$ & 0.514 \\
$\omega$ & $\varphi_{\omega}=0.010503517984676$ & 0.015 \\
$\gamma$ & $\varphi_{\gamma}=-0.011904372178292$ & 0.1 \\
$\alpha$ & $\varphi_{\alpha}=-0.047600000$ & 0.01 \\
$\lambda$ & $\varphi_{\lambda}=-0.952378458056419$ & 0.2 \\
$\eta$ & $\varphi_{\eta}=1.100 \times 10^{-6}$ & 0.5 \\
\hline
\end{tabular}

C. Uncertainty analysis for $R_{0}$

The five parameters out of 9 parameters have been chosen for uncertainty analysis because of uncertainties in their estimation [18]. In order to examine the uncertainty in the 
values of these parameters, each parameter is assumed to be a random variable with a corresponding probability density function. Latin hypercube sampling has been used to sample the five parameters that considered for uncertainty analysis. The other parameters are given in Table 1.

The five parameters $\eta, \mu, \beta_{1}, \theta, \gamma$ follow the following probability distributions:

$\beta_{1}$ follows normal distribution with mean and standard deviation 0.514 and 0.1 respectively. $\eta$ follows the uniform distribution on $[0,20]$.

Vaccine rate $\theta$ follows triangular distribution with minimum, maximum and mode as $0.001,0.002,0.003$.

The vaccine efficacy $\gamma$ follows the triangular distribution with minimum, maximum and mode as $0.05,0.1,0.15$.

The mean duration of latency $\eta$ follows the gamma distribution with mean 3 days and stand deviation 0.1 days.

Duration of infectious period $\lambda-1$ follows gamma distribution with mean 5days and standard deviation 1day.

Histograms for the distributions of the basic reproduction number types of parameter dis- tribution as shown in Figure 2.A set of 1000 parameter values has been also sampled from Latin hypercube sampling again from various different types of parameter distribution as shown in Figure 2.

Histograms for the distributions of the basic reproduction number are shown in Figure 3 and 4.These histograms have been generated from (2.1) using Latin hypercube sampling with sizes 1000 .
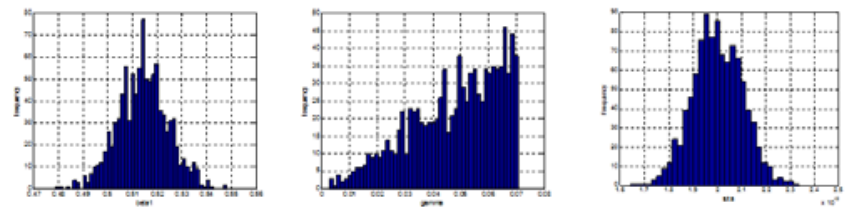

Histogram of the value of $\beta_{1}, \gamma, \theta$
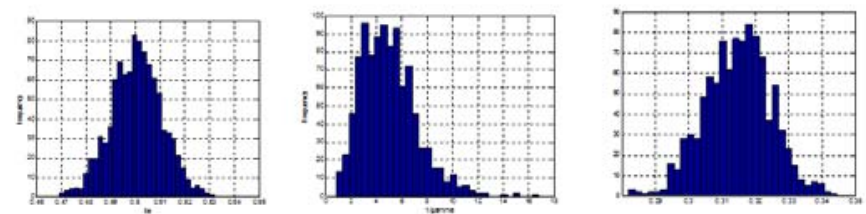

Histogram of the value of $\eta, \lambda, R_{0}$

FIGURE III. HISTOGRAMS OBTAINED FROM LATIN HYPERCUBE SAMPLING USING A SAMPLE OF 1000 FOR THE SIX INPUT PARAMETERS

Scatter-plots comparing the basic reproduction number against each of five parameters $\eta, \mu, \beta_{1}, \theta, \gamma$ are shown in Fig.4 for random sampling with sample size 1000. These scatter-plots clearly show the linear relationships (monotonicity) between outcome of $R_{0}$ and input parameters. And they verify the correctness of the theory.

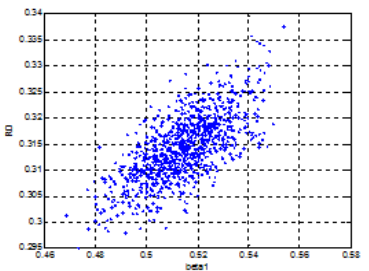

(a) $\beta_{1}$

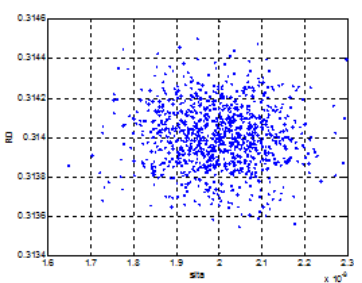

(c) $\theta$

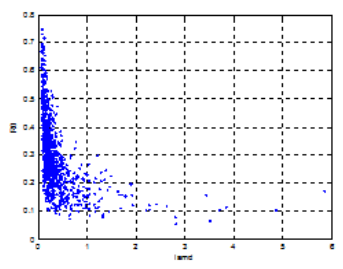

(e) $\lambda$

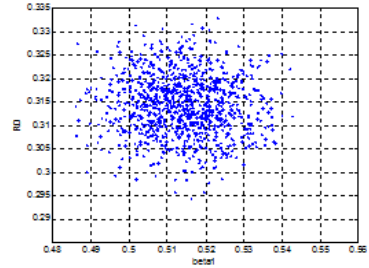

(b) $\gamma$

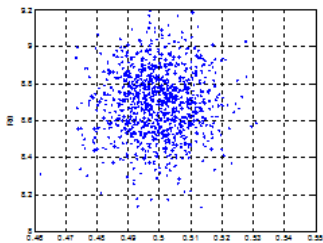

(d) $\eta$

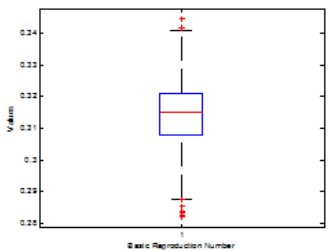

(f) Box Plot of the estimated $R_{0}$

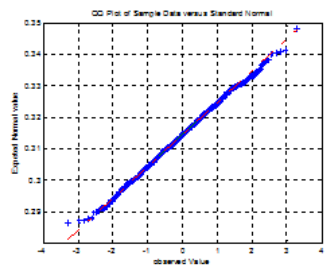

(g) Normal Q-Q Plot of the estimated $\lambda$ values FIGURE IV. GRAPHIC REPRESENTATION OF BASIC REPRODUCTION NUMBER BASED ON RANDOM SAMPLING

Box plots for the distributions of the basic reproduction number $R_{0}$ are shown in Fig. 4f. These Box plots have also been generated from system (2.1) using random sampling with sample sizes 1000. As shown in Fig.4 (f), the shaded box represents the first quartile, median and third quartile, which are quite symmetrically arranged. Few value are beyond the adjacent maximum line. However there are many values beyond the adjacent minimum line. The normal quantilequantile (Q-Q) plot and detrended Q-Q plot of the value of $R 0$ with sample sizes 1000 , using random sampling are shown in Fig.4g. It is shown that the observed values closely fit the expected normal values. The actual deviations of data points from the normal values have been shown in Fig $4 \mathrm{~g}$.

\section{The Impact of the Media Coverage}

In this section, we analyse the impact of media coverage on disease transmission. The impact of media coverage on the contact transmission $m_{I}$ in this paper $m_{I}<0$ means that thenegative effect caused by the contact media. The derivative 
of $\frac{I}{m_{I}+I}$ on $m_{I}$ is $-\frac{I}{\left(m_{I}+I\right)^{2}}<0$, so we can conclude that with the increase of $m_{I}, \beta_{1}-\beta_{2} \frac{I}{m_{I}+I}$ and $\beta_{1}-\beta_{3}$ $\frac{I}{m_{I}+I}$ will be more and more larger.

From Figure 5 we can see the effect of $m_{I}$ on infected communication. Inhibition of media coverage to the spread of disease is not endless, which is worth noting. Here, when $m_{I}$ $<-1$ and $m_{I}>0$, the disease transmission will hardly have any change. From Figure 5 we can see that values of $m_{I}$ is -0.9 , $0.6,-0.3,-0.1$ respectively, with the increase of $m_{I}$, the peak arrival will be delayed, and the peak value will also be bigger. If the $m_{I}$ is greater than 0 , then the infected will never change. So we can conclude that the media coverage is not a panacea, only can control the disease to a certain extent, but it have to be just perfect! Otherwise it will run counter to one's desire or harass the people and waste money!

\section{CONCLUSION}

For a long period of time $\mathrm{R}_{0}<1$ was considered the key to understand the dynamics of epidemiological models. In many cases, $\mathrm{R}_{0}<1$ will signify the disease free equilibrium is stable. And our control strategy is focused on how to effectively eliminate the disease. So from our view, we make some study on the sensitivity and uncertainty on the basic reproduction is very useful. On the other hand we also pay some attention to the influence of disease transmission media. In addition to other efforts, the emergence of H7N9 in China did not cause large-scale epidemic, which make us have to admit the great effects of media broadcasting.

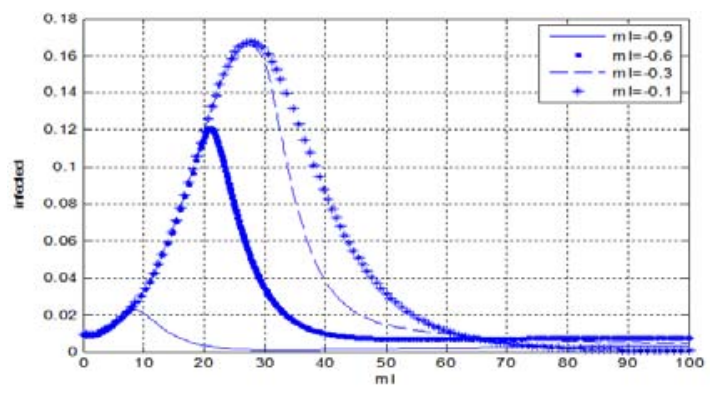

FIGURE V. THE IMPACT OF THE MEDIA COVERAGE

In this we formulated a model on the spread of influenza. Through mathematical method we worked out the disease-free equilibrium and basic reproductive number and analyzed the sensitivity and uncertainty of the basic reproductive number. We also make some simulation on the stability of the diseasefree equilibrium and the influence of the media coverage on the spread of the disease.

Our work award was conducive to more in-depth understanding of control the spread of the disease. And a better control of all the variables in the realistic society, the output of infectious disease effectively, so as serious errors can not be caused in terms of disease control.

\section{ACKNOWLEDGMENT}

Fund assistance: Our work was founded by "Case teaching model of differential equation based on mathematical modeling(JY2017B10)".

\section{REFERENCES}

[1] Liu R, Wu J, Zhu H. Media/psychological impact on multiple outbreak of emerging infectious disease.Comput. Meth. Med. 2007, 8(3):153-164.

[2] Y. Deguchi,Y. Tagasugi, Efficacy of influenza vaccine in the elderly: reduction in risk of mortality and mor- bidity during an influenza $\mathrm{A}(\mathrm{H} 3 \mathrm{~N} 2)$ epidemic for the elderly in nursing homes,Int.J.Clin.Lab.Res.30(2000)1- 4.

[3] D.J.D. Earn, J. Dushoff, S.A. Levin, Ecology and evolution of the flu, Trends Ecol. Evol.17(2002)334-340.

[4] J. Lin,V. Andreasen, S.A. Levin, Dynamics of influenza Adrift: the linear three-strain model,J.Math.Biosci.162(1999)33-51

[5] R.J. Webby, R.G. Webster, Are we ready for pandemic influenza?, Science 3202(2003)1519-1522.

[6] J.M. Tchuenche, N.DUBE, C. P. Bhunu, R. J. Smith, C. T. Bauch, Tchuenche et al: The impact of media coverage on the transmission dynamics of human influenza. BMC, Public Health 2011, 11 (Suppl 1) S5.

[7] Md. Samsuzzoha, M.Singh, D. Lucy, Uncertainty and sensitivity analysis of the basic reproduction number of a vaccinated epidemic model of influenza, Md. Samsuzzoha et al./Applied Mathematical Modelling 37(2013)903-915.

[8] Ma R: Media, Crisis, and SARS: An Introduction. Asian Journal of Communication 2005, 15(3):241-246. [9] Simpson CR: Nature as news: Science Reporting in The New York Times 1898 to 1983. The international journal of Politica, Culture and Society 1987, 1(2):218-241.

[9] Slater MD, Rasinski KA: Media Exposure and Attention as Mediating Variables Influencing Social Risk Judgments. Journal of Communication 2005, 55(4):810-827.

[10] Liu YCui J: The impact of media coverage on the dynamics of infectious disease. Int. J. Biomath. 2008 (1): 65-74.

[11] Cui JSun Y, Zhu H: The impact of media on the spreading and control of infectious disease. J. Dynam. Diff. Eqns. 2003(20):31-53.

[12] Cappasso V, Serio G: A generalization of the KermackMcKendrick deterministic epidemic model. Math.Biosci.1978, 42: 4362

[13] Cui J, Tao X, Zhu H: An SIS infection model incorporating media coverage. Rocky Mountain J. Math 2008, 38(5):1323-1334.

[14] Van den Driessche P, Watmough J:Reprodution numbers and subthreshold endemic equilibrium for com- partmental models of disease transmission. Math. Biosic. 2002(180): 29-48.

[15] Castillo-Chavez C, Feng Z Huang W: On the computation of R0 and its role on global stability. In Mathe- matical Approaches for Emerging and Reemerging Infectious Diseases: An Introduction. Berlin-HeidelbergNew York: Spring-Verlag; Castillo-Chavez C, vanden Driessche P, Kirschner D, Yakubu AA 2002:229-250.

[16] A. Hoare, D. G. Regan, D.P. Wilson, Sampling and sensitivity analyses tools (SaSAT) for computational modeling. Theor. Biol. Med. Model. 5(2008) 4, http://dx.doi.org/10.1186/1742-4682-5-4.

[17] C. van den Dool, M.J.M.Bonten, E. Hak, J. Wallinga, Modeling the effects of influenza vaccination of health care workers in hospital departments, Vaccine 27(44)(2009)6261-6267.

[18] M. Samsuzzoha, M. Singh, D. Lucy, A numerical study on an influenza epidemic model with vaccination and diffusion, submitted for publication. 